\title{
The effect of density stratification on the transverse oscillations of two parallel coronal loops
}

\author{
D. Robertson, M. S. Ruderman, and Y. Taroyan
}

\begin{abstract}
Department of Applied Mathematics, University of Sheffield, Hicks Building, Hounsfied Road, Sheffield, S3 7RH, UK e-mail: [D.Robertson;m.s.ruderman;Y.Taroyan]@sheffield.ac.uk
\end{abstract}

Received 13 January 2010 / Accepted 2 March 2010

\section{ABSTRACT}

\begin{abstract}
Transverse oscillations of coronal magnetic loops are routinely observed during the space missions. Since the first observation these oscillations were interpreted in terms of kink oscillations of magnetic tubes. Sometimes collective oscillations of two or more coronal loops are observed. This makes the development of theory of collective oscillations of a few parallel magnetic tubes desirable. Another reason for the development of this theory is that there are evidences that at least some coronal loops are not monolithic but consist of many thin magnetic threads. In this paper the linear theory of kink oscillations of two parallel magnetic tubes with the density varying along the tubes is developed. The asymptotic method is used to derive the system of equations governing the collective kink oscillations of two tubes. This system is used to study the effect of density variation on the eigenfrequencies of collective oscillations. The implication of the obtained results on coronal seismology is discussed.
\end{abstract}

Key words. magnetohydrodynamics (MHD) - methods: analytical - Sun: corona - Sun: oscillations - waves

\section{Introduction}

The solar corona is a highly non-uniform medium. Its structure is closely related to the magnetic field. In particular, in active regions of the corona there are myriads of coronal magnetic loops, which are the structures elongated in the magnetic field direction with the enhanced plasma temperature and density inside. These structures support many different types of oscillations. One of the particularly important types of coronal loop oscillations are transverse oscillations that were first observed by TRACE on 14 July 1998. The results of this observation were reported by Aschwanden et al. (1999) and Nakariakov et al. (1999), who interpreted these oscillations as standing fast kink waves in a magnetic flux tube.

After this first observation the transverse oscillations of coronal loops remain in the focus of theoretical studies. First theoretical interpretation of the observed transverse coronal loop oscillations were based on the simplest model of a coronal loop. In this model a coronal loop was represented by a straight homogeneous magnetic tube with the magnetic field lines frozen in the dense photospheric plasma at the foot points. Then the theory of oscillations of homogeneous magnetic tubes was used (e.g. Edwin \& Roberts 1983). Later theorists started to develop more sophisticated models that took such effects as the loop curvature, non-circular cross-section and magnetic twist into account. For the review of the recent progress in the theory of the transverse coronal loop oscillations see, e.g., a review by Ruderman \& Erdélyi (2009).

One particular and very important property of magnetic loops is that the plasma density inside the loop varies along the loop due to gravitational stratification. The theory of transverse oscillations of stratified coronal loops was developed by, e.g., Andries et al. (2005a); Diaz et al. (2006) and Dymova \& Ruderman (2006a). This theory was then successfully applied to coronal seismology (e.g., Andries et al. 2005b;
Dymova \& Ruderman 2006b; Van Doorsselaere et al. 2007; McEwan et al. 2008; Morton et al. 2009; see also a review by Andries et al. 2009).

Sometimes it is observed that a few neighboring coronal loops oscillate simultaneously. When the loops are close enough to each other the observed oscillations should be considered as collective oscillations of loop arrays. It is natural to start studying collective oscillations of loop arrays from studying oscillations of just two loops. This problem was first addressed by Luna et al. (2008) numerically and then by Van Doorsselaere et al. (2008) analytically in the thin tube approximation. This work was extended to studying collective oscillations of four loops by Ofman (2005) and multi-loop systems by Luna et al. (2009).

In this paper we aim to extend the theory of collective oscillations of two loops in another direction. We will take the density variation along the loops into account. The paper is organized as follows. In the next section we describe the equilibrium state and main assumptions, and present the governing equations. In Sect. 3 we introduce bicylindrical coordinates used in our analysis. In Sect. 4 we derive the system of equations governing the kink oscillations of two parallel loops. In Sect. 5 we re-derive the expressions for the frequencies of kink oscillations of two parallel homogeneous tubes previously obtained by Van Doorsselaere et al. (2008). In Sect. 6 we investigate the effect of density variation along the loops on the frequencies of kink oscillations. In Sect. 7 we study the implication of the obtained results on the coronal seismology. Section 8 contains the summary of our results and conclusions.

\section{Problem formulation}

We start our analysis from describing the equilibrium state. In this state there are two curved parallel coronal loops. They are situated in parallel vertical planes, and the lines connecting their axes at foot points are perpendicular to these planes (see Fig. 1). 


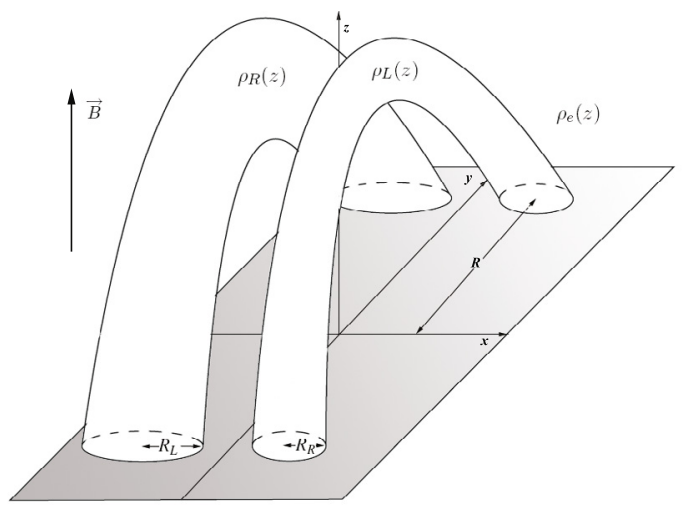

Fig. 1. Equilibrium with two parallel coronal magnetic loops.

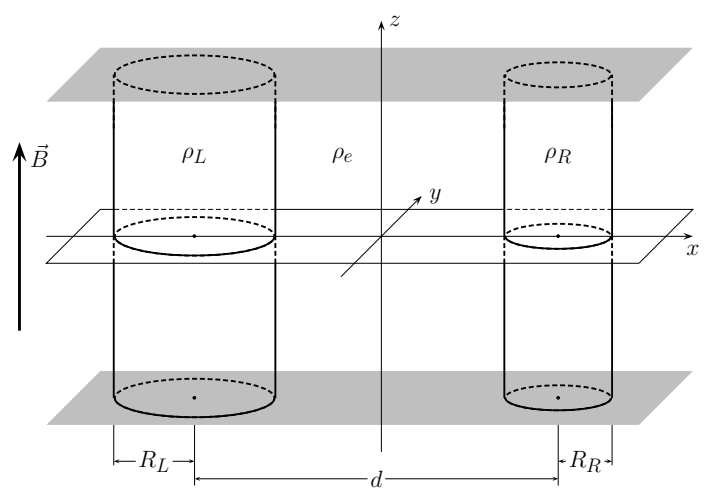

Fig. 2. The sketch of the equilibrium state with straight magnetic tubes. The magnetic filed lines are assumed to be frozen in a dense photospheric plasma at $z= \pm L / 2$. The equilibrium densities inside the tubes, $\rho_{\mathrm{L}}$ and $\rho_{\mathrm{R}}$, and outside the tubes, $\rho_{\mathrm{e}}$, are the functions of $z$.

The plasma density varies along the loops due to gravitational stratification.

In what follows we neglect the loop curvature. As a result we obtain a system of two straight parallel magnetic tubes with the density varying along the tubes. This system is shown in Fig. 2. The equilibrium magnetic field is in the $z$-direction, which coincides with the direction of the tube axes, and it has constant magnitude. The radii of the tubes are $R_{\mathrm{L}}$ and $R_{\mathrm{R}}$, and the distance between their axes is $d$. The density is equal to $\rho_{\mathrm{L}}(z)$ inside the left tube, $\rho_{\mathrm{R}}(z)$ inside the right tube, and $\rho_{\mathrm{e}}(z)$ outside the tubes. It is assumed that $\rho_{\mathrm{L}, \mathrm{R}}(z)>\rho_{\mathrm{e}}(z)$.

The plasma motion is described by the linearized ideal MHD equations for cold plasmas,

$\frac{\partial^{2} \boldsymbol{\xi}}{\partial t^{2}}=\frac{1}{\mu_{0} \rho}(\nabla \times \boldsymbol{b}) \times \boldsymbol{B}$,

$\boldsymbol{b}=\nabla \times(\boldsymbol{\xi} \times \boldsymbol{B})$.

Here $\boldsymbol{B}=\boldsymbol{B} \boldsymbol{e}_{z}$ and $\rho$ are the equilibrium magnetic field and density, $\boldsymbol{\xi}$ is the plasma displacement, $\boldsymbol{b}$ the magnetic field perturbation, $\boldsymbol{e}_{z}$ the unit vector in the $z$-direction, and $\mu_{0}$ the magnetic permeability of free space. The condition that the magnetic field lines are frozen in the dense photospheric plasma is written as

$\xi=0 \quad$ at $\quad z= \pm L / 2$.

The normal component of the plasma displacement, $\xi_{n}$, and the perturbation of the magnetic pressure, $P=B b_{z} / \mu_{0}$, have to be continuous at the tube boundaries. Finally, all the perturbations have to vanish far from the tubes.

\section{Introducing bicylindrical coordinates}

To study the oscillations of two-tube system we use bicylindrical coordinates $\tau, \sigma, z$, where $\tau$ varies from $-\infty$ to $\infty$, and $\sigma$ varies from 0 to $2 \pi$ (e.g. Korn \& Korn 1961). The coordinate lines in the $\tau \sigma$-plane are shown in Van Doorsselaere et al. (2008). The Cartesian and bicylindrical coordinates are related by

$x=\frac{a \sinh \tau}{\cosh \tau-\cos \sigma}, \quad y=\frac{a \sin \sigma}{\cosh \tau-\cos \sigma}, \quad z=z$,

where $a$ is a constant with the dimension of length. The axes of the tubes are parallel to the $z$-axis. The axes of the left and right tube cross the $x y$-plane at points $x=-d / 2$ and $x=d / 2$ respectively on the $x$-axis. The boundaries of the left and right tube are defined by $\tau=-\tau_{\mathrm{L}}$ and $\tau=\tau_{\mathrm{R}}$ respectively. The radii of tubes and the distance between their axes are related to $\tau_{\mathrm{L}}$ and $\tau_{\mathrm{R}}$ by

$R_{\mathrm{L}}=\frac{a}{\sinh \tau_{\mathrm{L}}}, \quad R_{\mathrm{R}}=\frac{a}{\sinh \tau_{\mathrm{R}}}, \quad d=a\left(\operatorname{coth} \tau_{\mathrm{L}}+\operatorname{coth} \tau_{\mathrm{R}}\right)$

It is shown by Van Doorsselaere et al. (2008) that $a, \tau_{\mathrm{L}}$ and $\tau_{\mathrm{R}}$ are uniquely defined for given $R_{\mathrm{L}}, R_{\mathrm{R}}$ and $d$ when $R_{\mathrm{L}}+R_{\mathrm{R}}<d$. In what follows we also use the expressions for the gradient and Laplacian in bicylindrical coordinates,

$\nabla f=\frac{\cosh \tau-\cos \sigma}{a}\left(\boldsymbol{e}_{\tau} \frac{\partial f}{\partial \tau}+\boldsymbol{e}_{\sigma} \frac{\partial f}{\partial \sigma}\right)+\boldsymbol{e}_{z} \frac{\partial f}{\partial z}$,

$\nabla^{2} f=\frac{(\cosh \tau-\cos \sigma)^{2}}{a^{2}}\left(\frac{\partial^{2} f}{\partial \tau^{2}}+\frac{\partial^{2} f}{\partial \sigma^{2}}\right)+\frac{\partial^{2} f}{\partial z^{2}}$.

Here $f$ is an arbitrary function, and $\boldsymbol{e}_{\tau}, \boldsymbol{e}_{\sigma}$ and $\boldsymbol{e}_{z}$ are the unit vectors in the $\tau, \sigma$ and $z$ directions respectively.

It follows from Eq. (1) that $\xi_{z}=0$. Then we obtain from Eq. (2) that

$P=-\rho V_{\mathrm{A}}^{2} \nabla \cdot \xi$,

where the square of the Alfvén speed is given by

$V_{\mathrm{A}}^{2}=\frac{B^{2}}{\mu_{0} \rho}$.

Equation (1) can be transformed to

$\frac{\partial^{2} \boldsymbol{\xi}}{\partial t^{2}}=\frac{V_{\mathrm{A}}^{2}}{B} \frac{\partial \boldsymbol{b}_{\perp}}{\partial z}-\frac{1}{\rho} \nabla_{\perp} P$,

where

$\boldsymbol{b}_{\perp}=\boldsymbol{b}-b_{z} \boldsymbol{e}_{z}, \quad \nabla_{\perp}=\nabla-\boldsymbol{e}_{z} \frac{\partial}{\partial z}$.

It follows from Eq. (2) that

$\boldsymbol{b}_{\perp}=B \frac{\partial \boldsymbol{\xi}}{\partial z}$

Substituting this result in Eq. (10) we obtain

$\frac{\partial^{2} \boldsymbol{\xi}}{\partial t^{2}}-V_{\mathrm{A}}^{2} \frac{\partial^{2} \boldsymbol{\xi}}{\partial z^{2}}=-\frac{1}{\rho} \nabla_{\perp} P$

Taking the divergence of this equation and using (8) yields

$\frac{\partial^{2} P}{\partial t^{2}}-V_{\mathrm{A}}^{2} \nabla P=0$ 
The conditions that the magnetic pressure perturbation and normal component of displacement are continuous at the tube boundaries are written as

$[P]=0, \quad\left[\xi_{\tau}\right]=0, \quad$ at $\quad \tau=-\tau_{\mathrm{L}}, \tau=\tau_{\mathrm{R}}$,

where the square brackets indicate the jump of a quantity across the boundary. It follows from Eqs. (3) and (8) that $P$ satisfies the boundary conditions

$P=0 \quad$ at $\quad z= \pm L / 2$.

Since $x \rightarrow \pm a$ and $y \rightarrow 0$ as $\tau \rightarrow \pm \infty, P$ and $\xi$ have to tend to finite limits as $\tau \rightarrow \pm \infty$. It is straightforward to show that $\tau \rightarrow 0$ and $\sigma \rightarrow 0$ as $x^{2}+y^{2} \rightarrow \infty$. Hence, we also impose the condition that $P \rightarrow 0$ and $\xi \rightarrow 0$ as $\tau^{2}+\sigma^{2} \rightarrow 0$.

Equations (13) and (14) together with the boundary conditions (15) and the limiting conditions as $\tau \rightarrow \pm \infty$ and $\tau^{2}+\sigma^{2} \rightarrow$ 0 are used in the next section to derive the governing equations for the tube oscillations.

\section{Derivation of governing equations}

In this section we derive the system of two equations governing the kink oscillations of two parallel magnetic tubes in the thin tube approximation. To do this we use the same asymptotic method that was used by Dymova \& Ruderman (2005) to derive the governing equation for a single stratified tube, and by Van Doorsselaere et al. (2008) to derive the dispersion relation for kink oscillations of two parallel homogeneous tubes. To use this method we assume that the characteristic spatial scale of the two-tube system in the transverse direction is much smaller than $L$. We can take this characteristic scale to be equal to $d$, so that we assume that $\epsilon=d / L \ll 1$. Since the characteristic scale in the $z$-direction is much larger than $d$, we introduce the stretching variable $Z=\epsilon z$. We consider oscillations with periods of the order of $L / V_{\mathrm{A}}^{*}$, where $V_{\mathrm{A}}^{*}$ is the characteristic value of the Alfvén speed. The quantity $L / V_{\mathrm{A}}^{*}$ can be considered as the characteristic time of the problem. Since $L / V_{\mathrm{A}}^{*}=\epsilon^{-1} d / V_{\mathrm{A}}^{*}$, where $d / V_{\mathrm{A}}^{*}$ is the Alfvénic time in the transverse direction, we also introduce the "slow" time $T=\epsilon t$. In the new variables the Eqs. (13) and (14) are transformed to

$\frac{\partial^{2} \xi_{\tau}}{\partial T^{2}}-V_{\mathrm{A}}^{2} \frac{\partial^{2} \xi_{\tau}}{\partial Z^{2}}=-\frac{\cosh \tau-\cos \sigma}{\epsilon^{2} a \rho} \frac{\partial P}{\partial \tau}$,

$\frac{\partial^{2} \xi_{\sigma}}{\partial T^{2}}-V_{\mathrm{A}}^{2} \frac{\partial^{2} \xi_{\sigma}}{\partial Z^{2}}=-\frac{\cosh \tau-\cos \sigma}{\epsilon^{2} a \rho} \frac{\partial P}{\partial \sigma}$

$\epsilon^{2} \frac{\partial^{2} P}{\partial T^{2}}-\epsilon^{2} V_{\mathrm{A}}^{2} \frac{\partial^{2} P}{\partial Z^{2}}-V_{\mathrm{A}}^{2} \nabla_{\perp}^{2} P=0$.

Neglecting terms of the order of $\epsilon^{2}$ in Eq. (19) and using Eqs. (7) and (11) we obtain the approximate equation

$\frac{\partial^{2} P}{\partial \tau^{2}}+\frac{\partial^{2} P}{\partial \sigma^{2}}=0$

The general solution to this equation can be looked for in the form of Fourier expansion with respect to $\sigma$. However we are only interested in the kink oscillations, so that we look for the solution to Eq. (20) in the form

$P=\Phi(\tau, T, Z) \cos \left(\sigma-\sigma_{0}\right)-\Phi(0, T, Z) \cos \sigma_{0}$.
The second term on the right-hand side of this expression is introduced to satisfy the condition that $P \rightarrow 0$ as $\tau^{2}+\sigma^{2} \rightarrow 0$. Now we easily find that $P$ satisfies Eq. (20), the conditions that $P$ tends to finite limits when $\tau \rightarrow \pm \infty$, and is continuous at the tube boundaries, if $\Phi$ is given by

$\Phi(\tau, T, Z)= \begin{cases}\left(C_{1}+C_{2} \mathrm{e}^{2 \tau_{\mathrm{L}}}\right) \mathrm{e}^{\tau}, & \tau<-\tau_{\mathrm{L}}, \\ C_{1} \mathrm{e}^{\tau}+C_{2} \mathrm{e}^{-\tau}, & -\tau_{\mathrm{L}}<\tau<\tau_{\mathrm{R}}, \\ \left(C_{1} \mathrm{e}^{2 \tau_{\mathrm{R}}}+C_{2}\right) \mathrm{e}^{-\tau}, & \tau>\tau_{\mathrm{R}},\end{cases}$

where $C_{1}$ and $C_{2}$ are arbitrary functions of $T$ and $Z$ satisfying $C_{1}(T, \pm \epsilon L / 2)=C_{2}(T, \pm \epsilon L / 2)=0$.

Substituting the expression for $P$ in Eqs. (17) and (18) we transform them to

$\frac{\partial^{2} \xi_{\tau}}{\partial T^{2}}-V_{\mathrm{A}}^{2} \frac{\partial^{2} \xi_{\tau}}{\partial Z^{2}}=-\frac{Q(\tau, \sigma)}{\rho} \frac{\partial \Phi}{\partial \tau} \cos \left(\sigma-\sigma_{0}\right)$,

$\frac{\partial^{2} \xi_{\sigma}}{\partial T^{2}}-V_{\mathrm{A}}^{2} \frac{\partial^{2} \xi_{\sigma}}{\partial Z^{2}}=\frac{Q(\tau, \sigma)}{\rho} \Phi(\tau) \sin \left(\sigma-\sigma_{0}\right)$,

where $Q(\tau, \sigma)$ is given by

$Q(\tau, \sigma)=\frac{\cosh \tau-\cos \sigma}{\epsilon^{2} a}$.

Using Eq. (22) we obtain that $\xi_{\tau}$ and $\xi_{\sigma}$ are defined by

$\frac{\partial^{2} \xi_{\tau}}{\partial T^{2}}-V_{\mathrm{AL}}^{2} \frac{\partial^{2} \xi_{\tau}}{\partial Z^{2}}=-\frac{Q}{\rho_{\mathrm{L}}}\left(C_{1}+C_{2} \mathrm{e}^{2 \tau_{\mathrm{L}}}\right) \mathrm{e}^{\tau} \cos \left(\sigma-\sigma_{0}\right)$

$\frac{\partial^{2} \xi_{\sigma}}{\partial T^{2}}-V_{\mathrm{AL}}^{2} \frac{\partial^{2} \xi_{\sigma}}{\partial Z^{2}}=\frac{Q}{\rho_{\mathrm{L}}}\left(C_{1}+C_{2} \mathrm{e}^{2 \tau_{\mathrm{L}}}\right) \mathrm{e}^{\tau} \sin \left(\sigma-\sigma_{0}\right)$,

for $\tau<-\tau_{\mathrm{L}}$, by

$\frac{\partial^{2} \xi_{\tau}}{\partial T^{2}}-V_{\mathrm{Ae}}^{2} \frac{\partial^{2} \xi_{\tau}}{\partial Z^{2}}=\frac{Q}{\rho_{\mathrm{e}}}\left(-C_{1} \mathrm{e}^{\tau}+C_{2} \mathrm{e}^{-\tau}\right) \cos \left(\sigma-\sigma_{0}\right)$,

$\frac{\partial^{2} \xi_{\sigma}}{\partial T^{2}}-V_{\mathrm{Ae}}^{2} \frac{\partial^{2} \xi_{\sigma}}{\partial Z^{2}}=\frac{Q}{\rho_{\mathrm{e}}}\left(C_{1} \mathrm{e}^{\tau}+C_{2} \mathrm{e}^{-\tau}\right) \sin \left(\sigma-\sigma_{0}\right)$,

for $-\tau_{\mathrm{L}}<\tau<\tau_{\mathrm{R}}$, and by

$\frac{\partial^{2} \xi_{\tau}}{\partial T^{2}}-V_{\mathrm{AR}}^{2} \frac{\partial^{2} \xi_{\tau}}{\partial Z^{2}}=\frac{Q}{\rho_{\mathrm{R}}}\left(C_{1} \mathrm{e}^{2 \tau_{\mathrm{R}}}+C_{2}\right) \mathrm{e}^{-\tau} \cos \left(\sigma-\sigma_{0}\right)$,

$\frac{\partial^{2} \xi_{\sigma}}{\partial T^{2}}-V_{\mathrm{AR}}^{2} \frac{\partial^{2} \xi_{\sigma}}{\partial Z^{2}}=\frac{Q}{\rho_{\mathrm{R}}}\left(C_{1} \mathrm{e}^{2 \tau_{\mathrm{R}}}+C_{2}\right) \mathrm{e}^{-\tau} \sin \left(\sigma-\sigma_{0}\right)$,

for $\tau>\tau_{\mathrm{R}}$.

Let us introduce functions $\eta_{\mathrm{L}}(T, Z), \eta_{1}(T, Z), \eta_{2}(T, Z)$ and $\eta_{\mathrm{R}}(T, Z)$ satisfying

$\frac{\partial^{2} \eta_{\mathrm{L}}}{\partial T^{2}}-V_{\mathrm{AL}}^{2} \frac{\partial^{2} \eta_{\mathrm{L}}}{\partial Z^{2}}=\frac{\mathrm{e}^{\tau_{\mathrm{R}}}\left(C_{1}+C_{2} \mathrm{e}^{2 \tau_{\mathrm{L}}}\right)}{\rho_{\mathrm{L}}}$

$\frac{\partial^{2} \eta_{\mathrm{R}}}{\partial T^{2}}-V_{\mathrm{AR}}^{2} \frac{\partial^{2} \eta_{\mathrm{R}}}{\partial Z^{2}}=\frac{\mathrm{e}^{\tau_{\mathrm{L}}}\left(C_{1} \mathrm{e}^{2 \tau_{\mathrm{R}}}+C_{2}\right)}{\rho_{\mathrm{R}}}$,

$\frac{\partial^{2} \eta_{1,2}}{\partial T^{2}}-V_{\mathrm{Ae}}^{2} \frac{\partial^{2} \eta_{1,2}}{\partial Z^{2}}=\frac{C_{1,2}}{\rho_{\mathrm{e}}}$ 
Then it follows from Eqs. (26) and (27) that

$\xi_{\tau}=Q \cos \left(\sigma-\sigma_{0}\right)\left\{\begin{array}{cc}-\eta_{\mathrm{L}} \mathrm{e}^{\tau-\tau_{\mathrm{R}}}, & \tau<-\tau_{\mathrm{L}}, \\ -\eta_{1} \mathrm{e}^{\tau}+\eta_{2} \mathrm{e}^{-\tau}, & -\tau_{\mathrm{L}}<\tau<\tau_{\mathrm{R}}, \\ \eta_{\mathrm{R}} \mathrm{e}^{-\tau-\tau_{\mathrm{L}}}, & \tau>\tau_{\mathrm{R}},\end{array}\right.$

$\xi_{\sigma}=-Q \sin \left(\sigma-\sigma_{0}\right)\left\{\begin{array}{lc}\eta_{\mathrm{L}} \mathrm{e}^{\tau-\tau_{\mathrm{R}}}, & \tau<-\tau_{\mathrm{L}}, \\ \eta_{1} \mathrm{e}^{\tau}+\eta_{2} \mathrm{e}^{-\tau}, & -\tau_{\mathrm{L}}<\tau<\tau_{\mathrm{R}}, \\ \eta_{\mathrm{R}} \mathrm{e}^{-\tau-\tau_{\mathrm{L}}}, & \tau>\tau_{\mathrm{R}} .\end{array}\right.$

It follows from the second boundary condition in Eqs. (15) and (35) that

$E \eta_{\mathrm{L}}=\eta_{1} \mathrm{e}^{-\tau_{\mathrm{L}}}-\eta_{2} \mathrm{e}^{\tau_{\mathrm{L}}}, \quad E \eta_{\mathrm{R}}=-\eta_{1} \mathrm{e}^{\tau_{\mathrm{R}}}+\eta_{2} \mathrm{e}^{-\tau_{\mathrm{R}}}$,

where $E=\exp \left[-\left(\tau_{\mathrm{L}}+\tau_{\mathrm{R}}\right)\right]$. These equations imply that

$\eta_{1}=-\frac{\eta_{\mathrm{R}} \mathrm{e}^{\tau_{\mathrm{L}}}+\eta_{\mathrm{L}} \mathrm{e}^{-\tau_{\mathrm{R}}}}{E^{-2}-1}, \quad \eta_{2}=-\frac{\eta_{\mathrm{L}} \mathrm{e}^{\tau_{\mathrm{R}}}+\eta_{\mathrm{R}} \mathrm{e}^{-\tau_{\mathrm{L}}}}{E^{-2}-1}$.

Substituting these expressions in Eq. (34) we obtain

$$
\begin{aligned}
\mathrm{e}^{\tau_{\mathrm{L}}}\left(\frac{\partial^{2} \eta_{\mathrm{R}}}{\partial T^{2}}-V_{\mathrm{Ae}}^{2} \frac{\partial^{2} \eta_{\mathrm{R}}}{\partial Z^{2}}\right)+\mathrm{e}^{-\tau_{\mathrm{R}}}\left(\frac{\partial^{2} \eta_{\mathrm{L}}}{\partial T^{2}}\right. & \left.-V_{\mathrm{Ae}}^{2} \frac{\partial^{2} \eta_{\mathrm{L}}}{\partial Z^{2}}\right) \\
& =-\frac{C_{1}}{\rho_{\mathrm{e}}}\left(E^{-2}-1\right),
\end{aligned}
$$

$$
\begin{aligned}
\mathrm{e}^{\tau_{\mathrm{R}}}\left(\frac{\partial^{2} \eta_{\mathrm{L}}}{\partial T^{2}}-V_{\mathrm{Ae}}^{2} \frac{\partial^{2} \eta_{\mathrm{L}}}{\partial Z^{2}}\right)+\mathrm{e}^{-\tau_{\mathrm{L}}}\left(\frac{\partial^{2} \eta_{\mathrm{R}}}{\partial T^{2}}\right. & \left.-V_{\mathrm{Ae}}^{2} \frac{\partial^{2} \eta_{\mathrm{R}}}{\partial Z^{2}}\right) \\
& =-\frac{C_{2}}{\rho_{\mathrm{e}}}\left(E^{-2}-1\right) .
\end{aligned}
$$

Eliminating $C_{1}$ and $C_{2}$ from Eqs. (32), (33), (39) and (40), and returning to the original independent variables, we arrive at equations for $\eta_{\mathrm{L}}$ and $\eta_{\mathrm{R}}$,

$\frac{\partial^{2} \eta_{\mathrm{L}}}{\partial t^{2}}-C_{k \mathrm{~L}}^{2} \frac{\partial^{2} \eta_{\mathrm{L}}}{\partial z^{2}}=E \frac{\rho_{\mathrm{R}}-\rho_{\mathrm{e}}}{\rho_{\mathrm{L}}+\rho_{\mathrm{e}}} \frac{\partial^{2} \eta_{\mathrm{R}}}{\partial t^{2}}$,

$\frac{\partial^{2} \eta_{\mathrm{R}}}{\partial t^{2}}-C_{k \mathrm{R}}^{2} \frac{\partial^{2} \eta_{\mathrm{R}}}{\partial z^{2}}=E \frac{\rho_{\mathrm{L}}-\rho_{\mathrm{e}}}{\rho_{\mathrm{R}}+\rho_{\mathrm{e}}} \frac{\partial^{2} \eta_{\mathrm{L}}}{\partial t^{2}}$,

where the squares of the kink speeds for the left and right tubes are given by

$C_{k \mathrm{~L}}^{2}=\frac{2 \rho V_{\mathrm{A}}^{2}}{\rho_{\mathrm{L}}+\rho_{\mathrm{e}}}, \quad C_{k \mathrm{R}}^{2}=\frac{2 \rho V_{\mathrm{A}}^{2}}{\rho_{\mathrm{R}}+\rho_{\mathrm{e}}}$.

Let us introduce the dimensionless parameters

$r=\frac{R_{\mathrm{L}}+R_{\mathrm{R}}}{d}, \quad s=\frac{R_{\mathrm{R}}}{R_{\mathrm{L}}}$.

Obviously $r \leq 1$. Using Eq. (5) it is straightforward to obtain

$\cosh \tau_{\mathrm{L}}=\frac{1+s+r^{2}(1-s)}{2 r}, \cosh \tau_{\mathrm{R}}=\frac{1+s-r^{2}(1-s)}{2 r s}$.

These relations can be considered as quadratic equations for $\mathrm{e}^{-\tau_{\mathrm{L}}}$ and $\mathrm{e}^{-\tau_{\mathrm{R}}}$. Calculating this quantity we can then find the expression for $E=\exp \left[-\left(\tau_{\mathrm{L}}+\tau_{\mathrm{R}}\right)\right]$, and obtain the relation between $E$, $r$ and $s$. After long but straightforward calculation we arrive at

$r^{2}=\frac{E(1+s)^{2}}{(s+E)(1+s E)}$.
When $E$ is fixed, $r$ is a function of $s$. It is easy to show that $r<1$ for any value of $s>0, r \rightarrow 1$ when either $s \rightarrow 0$ or $s \rightarrow \infty$, and $r$ takes it minimum value $r_{\min }$ when $s=1$, where

$r_{\min }=\frac{2 \sqrt{E}}{1+E}$.

In the two-tube system the lines that are not perturbed by the fluting modes are not the tube axes, but the lines parallel to the tube axes and crossing the $x y$-plane at points with coordinates $(-a, 0)$ and $(a, 0)$. In bicylindrical coordinates these straight lines correspond to $\tau \rightarrow-\infty$ and $\tau \rightarrow \infty$ respectively. Let us denote the displacements of these lines as $\xi_{\mathrm{L}}$ and $\boldsymbol{\xi}_{\mathrm{R}}$. Using Eqs. (35) and (36) we obtain that

$$
\begin{aligned}
& \boldsymbol{\xi}_{\mathrm{L}}=\frac{\eta_{\mathrm{L}} \mathrm{e}^{-\tau_{\mathrm{R}}}}{2 \epsilon^{2} a}\left[-\boldsymbol{e}_{\tau} \cos \left(\sigma-\sigma_{0}\right)+\boldsymbol{e}_{\sigma} \sin \left(\sigma-\sigma_{0}\right)\right], \\
& \boldsymbol{\xi}_{\mathrm{R}}=\frac{\eta_{\mathrm{R}} \mathrm{e}^{-\tau_{\mathrm{L}}}}{2 \epsilon^{2} a}\left[\boldsymbol{e}_{\tau} \cos \left(\sigma-\sigma_{0}\right)+\boldsymbol{e}_{\sigma} \sin \left(\sigma-\sigma_{0}\right)\right] .
\end{aligned}
$$

Using the relations

$$
\begin{aligned}
& \boldsymbol{e}_{\tau}=\frac{\boldsymbol{e}_{x}(1-\cosh \tau \cos \sigma)-\boldsymbol{e}_{y} \sinh \tau \sin \sigma}{(\cosh \tau-\cos \sigma)} \\
& \boldsymbol{e}_{\sigma}=-\frac{\boldsymbol{e}_{x} \sinh \tau \sin \sigma+\boldsymbol{e}_{y}(1-\cosh \tau \cos \sigma)}{(\cosh \tau-\cos \sigma)},
\end{aligned}
$$

where $\boldsymbol{e}_{x}$ and $\boldsymbol{e}_{y}$ are the unit vectors in the $x$ and $y$-direction, we obtain that

$$
\begin{aligned}
& \boldsymbol{e}_{\tau} \rightarrow-\boldsymbol{e}_{x} \cos \sigma+\boldsymbol{e}_{y} \sin \sigma \\
& \boldsymbol{e}_{\sigma} \rightarrow \boldsymbol{e}_{x} \sin \sigma+\boldsymbol{e}_{y} \cos \sigma,
\end{aligned}
$$

as $\tau \rightarrow-\infty$, and

$$
\begin{aligned}
& \boldsymbol{e}_{\tau} \rightarrow-\boldsymbol{e}_{x} \cos \sigma-\boldsymbol{e}_{y} \sin \sigma, \\
& \boldsymbol{e}_{\sigma} \rightarrow-\boldsymbol{e}_{x} \sin \sigma+\boldsymbol{e}_{y} \cos \sigma,
\end{aligned}
$$

as $\tau \rightarrow \infty$. With the aid of these results we rewrite Eq. (48) as

$\boldsymbol{\xi}_{\mathrm{L}}=\frac{\eta_{\mathrm{L}} \mathrm{e}^{-\tau_{\mathrm{R}}}}{2 \epsilon^{2} a}\left(-\boldsymbol{e}_{x} \cos \sigma_{0}+\boldsymbol{e}_{y} \sin \sigma_{0}\right)$,

$\boldsymbol{\xi}_{\mathrm{R}}=\frac{\eta_{\mathrm{R}} \mathrm{e}^{-\tau_{\mathrm{L}}}}{2 \epsilon^{2} a}\left(\boldsymbol{e}_{x} \cos \sigma_{0}+\boldsymbol{e}_{y} \sin \sigma_{0}\right)$

These relations will be used in the discussion of polarization of eigenmodes.

\section{Eigenmodes of system with constant density}

Let us use Eqs. (41) and (42) to study the eigenmodes of the system of two parallel homogeneous tubes. We will only consider the fundamental mode with respect to $z$, and take $\eta_{\mathrm{L}}$ and $\eta_{\mathrm{R}}$ proportional to $\exp (-\mathrm{i} \omega) \cos (k z)$, where $k=\pi / L$. Then the system of Eqs. (41) and (42) reduces to

$\left(\omega^{2}-k^{2} C_{k \mathrm{~A}}^{2}\right) \eta_{\mathrm{L}}=E \frac{\rho_{\mathrm{R}}-\rho_{\mathrm{L}}}{\rho_{\mathrm{L}}+\rho_{\mathrm{e}}} \omega^{2} \eta_{\mathrm{R}}$

$\left(\omega^{2}-k^{2} C_{k \mathrm{~L}}^{2}\right) \eta_{\mathrm{R}}=E \frac{\rho_{\mathrm{L}}-\rho_{\mathrm{e}}}{\rho_{\mathrm{R}}+\rho_{\mathrm{e}}} \omega^{2} \eta_{\mathrm{L}}$

We obtained the system of two linear homogeneous algebraic equations for $\eta_{\mathrm{L}}$ and $\eta_{\mathrm{R}}$. This system has non-trivial solutions 


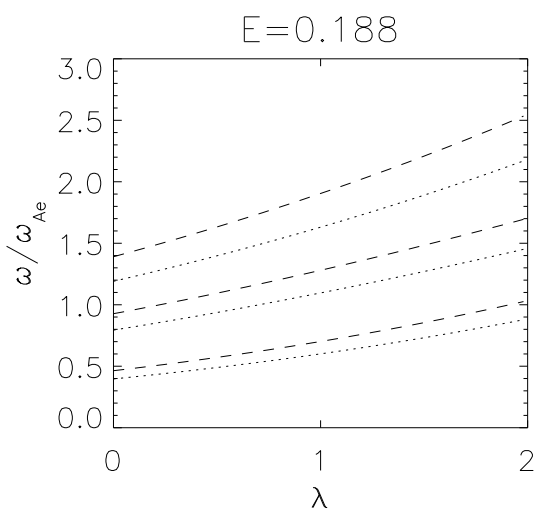

only when its determinant is zero. This condition gives the dispersion equation relating $\omega$ and $k$. The solutions of this dispersion equation are $\omega= \pm \omega_{+}$and $\omega= \pm \omega_{-}$, where

$\omega_{ \pm}^{2}=\frac{V_{\mathrm{Ae}}^{2} k^{2}\left(\zeta_{\mathrm{L}}+\zeta_{\mathrm{R}}+2 \pm Q\right)}{\left(\zeta_{\mathrm{L}}+1\right)\left(\zeta_{\mathrm{R}}+1\right)-\left(\zeta_{\mathrm{L}}-1\right)\left(\zeta_{\mathrm{R}}-1\right) E^{2}}$,

$\zeta_{\mathrm{L}}=\frac{\rho_{\mathrm{L}}}{\rho_{\mathrm{e}}}, \quad \zeta_{\mathrm{R}}=\frac{\rho_{\mathrm{R}}}{\rho_{\mathrm{e}}}$,

$Q=\sqrt{\left(\zeta_{\mathrm{L}}-\zeta_{\mathrm{R}}\right)^{2}+4\left(\zeta_{\mathrm{L}}-1\right)\left(\zeta_{\mathrm{R}}-1\right) E^{2}}$.

This result is in agreement with the result obtained by Van Doorsselaere et al. (2008). In what follows we will call the oscillation mode with the frequency $\omega_{+}$the high-frequency mode, and the mode with the frequency $\omega_{-}$the low-frequency mode.

\section{Effect of density stratification}

In this section we study the effect of density stratification on the eigenmodes of the two-tube system oscillations. We look for the solution to the system of Eqs. (41) and (42) with $\eta_{\mathrm{L}}$ and $\eta_{\mathrm{R}}$ proportional to $\exp (-\mathrm{i} \omega)$. The solution has to satisfy the boundary conditions

$\eta_{\mathrm{L}}=\eta_{\mathrm{R}}=0 \quad$ at $\quad z= \pm L / 2$.

Equations (41) and (42) are now reduced to

$\frac{d^{2} \eta_{\mathrm{L}}}{d z^{2}}+\frac{\omega^{2}}{C_{k \mathrm{~L}}^{2}}\left(\eta_{\mathrm{L}}-\frac{\zeta_{\mathrm{R}}-1}{\zeta_{\mathrm{L}}+1} E \eta_{\mathrm{R}}\right)=0$,

$\frac{d^{2} \eta_{\mathrm{R}}}{d z^{2}}+\frac{\omega^{2}}{C_{k \mathrm{R}}^{2}}\left(\eta_{\mathrm{R}}-\frac{\zeta_{\mathrm{L}}-1}{\zeta_{\mathrm{R}}+1} E \eta_{\mathrm{L}}\right)=0$.

Equations (59) and (60) together with the boundary condition (58) constitute the eigenvalue problem determining $\omega$. This eigenvalue problem was solved numerically. We assumed that both tubes have the shape of half-circle of radius $R$, so that the distance along the tube, $z$, is related to the atmospheric height, $h$, by $h=R \cos (z / R)$. We also assumed that the atmosphere is isothermal, and the temperature is the same inside the tubes and in the surrounding plasma, so that $\rho_{\mathrm{e}, \mathrm{L}, \mathrm{R}}=\bar{\rho}_{\mathrm{e}, \mathrm{L}, \mathrm{R}} \exp (-h / H)$, which can be rewritten as

$\rho_{\mathrm{e}, \mathrm{L}, \mathrm{R}}=\bar{\rho}_{\mathrm{e}, \mathrm{L}, \mathrm{R}} \exp \left(-\frac{R}{H} \cos \frac{z}{R}\right)$,
$E=1$

Fig. 3. Dependence of the normalized frequencies on $\lambda$ for $\zeta_{\mathrm{L}}=\zeta_{\mathrm{R}}=10$, and $E=0.188$ and $E=1$. The dashed and dotted curves correspond to the high-frequency and low-frequency eigenmodes. The frequencies of the fundamental mode, and the first and second overtones are shown. where $H$ is the atmospheric scale height, and $\bar{\rho}_{\mathrm{e}, \mathrm{L}, \mathrm{R}}$ denotes the densities at the foot points. Hence, $\zeta_{\mathrm{L}}$ and $\zeta_{\mathrm{R}}$ are constant, and the expressions for $C_{k \mathrm{~L}}^{2}$ and $C_{k \mathrm{R}}^{2}$ reduce to

$C_{k \mathrm{~L}, \mathrm{R}}^{2}=\frac{2 \bar{V}_{\mathrm{Ae}}^{2}}{\zeta_{\mathrm{L}, \mathrm{R}}+1} \exp \left(\frac{R}{H} \cos \frac{z}{R}\right)$,

where $\bar{V}_{\mathrm{Ae}}^{2}=B_{0}^{2} /\left(\mu_{0} \bar{\rho}_{\mathrm{e}}\right)$ is the square of the Alfvén speed in the surrounding plasma at the foot point level.

Figure 3 shows the dependence of frequencies of the highfrequency and low-frequency eigenmodes on the parameter $\lambda=$ $R / H$ for $\zeta_{\mathrm{L}}=\zeta_{\mathrm{R}}=10$. The left panel corresponds to $E=0.188$. When $s=2$ this corresponds to $r=3 / 4$, i.e. for tubes with the radius ratio of two the distance between the tube axes is twice the larger radius. The right panel corresponds to $E=1$. It follows from Eq. (46) that $r=1$ for any value of $s$, i.e. we have the limiting case when the tubes touch each other. In both panels the frequencies of the high-frequency eigenmodes are shown by dashed lines, and those of the low-frequency eigenmodes by dotted lines. The frequencies of the fundamental mode, and the first and second overtones are shown. The frequencies are normalized to the fundamental Alfvén frequency in the external plasma at the foot point level, $\omega_{\mathrm{Ae}}=k \bar{V}_{\mathrm{Ae}}$.

Figure 4 shows the dependence of frequencies of the highfrequency and low-frequency eigenmodes on $E$ for $\zeta_{\mathrm{L}}=\zeta_{\mathrm{R}}=$ 10. The left panel is for $\lambda=0$, which corresponds to nonstratified tubes. The right panel is for $\lambda=1$, which corresponds to $H=R$. If we want to find the values of frequencies for particular values of $r$ and $s$, then we need to calculate the corresponding value of $E$ using Eq. (46). For example, if we take $s=1$, i.e. $R_{\mathrm{L}}=R_{\mathrm{R}}$, and $r=4 / 5$, i.e. $d=\frac{5}{2} R_{\mathrm{L}}$, then we obtain $E=1 / 4$.

Figure 5 shows the dependence of frequencies of the highfrequency and low-frequency eigenmodes on the ratio of plasma densities outside and inside the tubes when the densities inside the tubes are the same, $\zeta_{\mathrm{L}}=\zeta_{\mathrm{R}}=\zeta$, and $E=7-3 \sqrt{5} \approx 0.292$. When the tube radii are equal $(s=1)$, this value of $E$ corresponds to $r=2 / 3$. The left panel is for $\lambda=0$, which corresponds to non-stratified tubes. The right panel is for $\lambda=1$, which corresponds to $H=R$.

Let us discuss the mode polarization. It is well known that the kink oscillations of straight magnetic tubes are infinitely degenerated in the long wavelength approximation in the sense that the oscillation frequency is independent of the polarization. Van Doorsselaere et al. (2008) showed that the same is true for the two-tube system when there is no density stratification. This property remains valid even when the tubes are stratified. Really, the eigenvalue problem determining the dependence of $\omega$ on $k$ does not contain the parameter $\sigma_{0}$, which determines the mode polarization. It follows from Eq. (52) that the vectors $\xi_{\mathrm{L}}$ and $\boldsymbol{\xi}_{\mathrm{R}}$ 

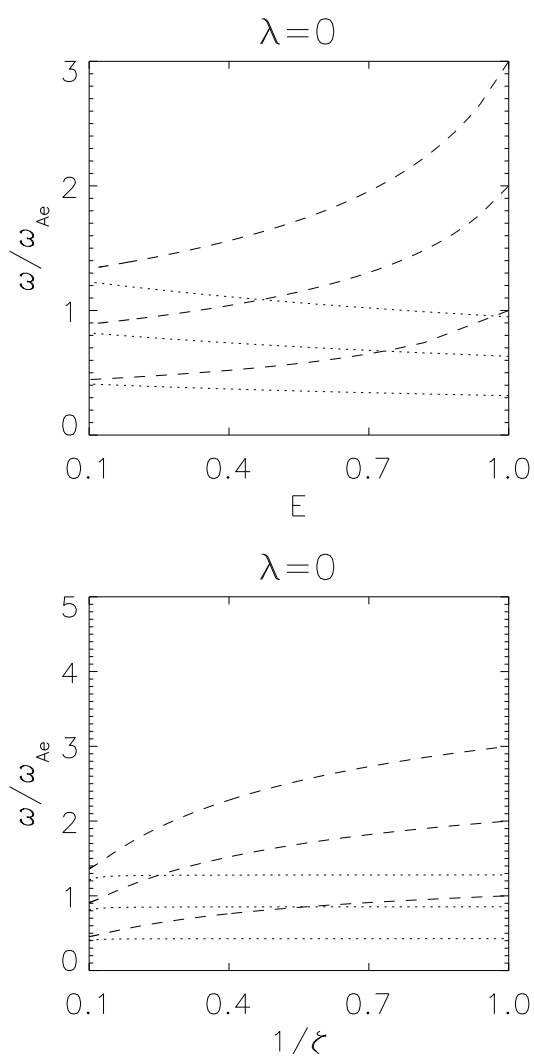
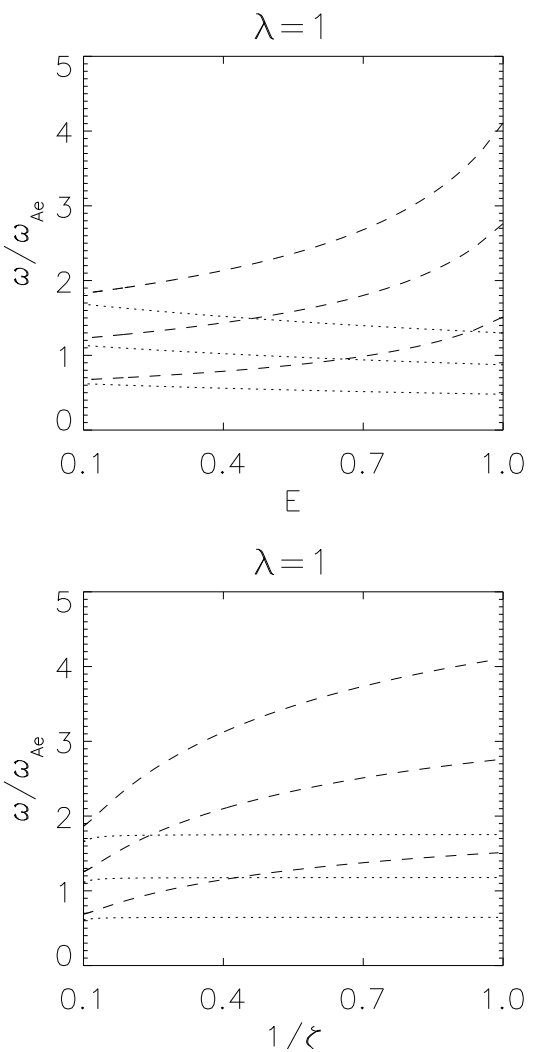

Fig. 4. Dependence of the normalized frequencies on $E$ for $\zeta_{\mathrm{L}}=\zeta_{\mathrm{R}}=10$. The left and right panels correspond to $\lambda=0$ and $\lambda=1$. The dashed and dotted curves correspond to the high-frequency and low-frequency eigenmodes. The frequencies of the fundamental mode, and the first and second overtones are shown. Note that, for fixed $R_{\mathrm{L}} / R_{\mathrm{R}}$, the decrease of $E$ corresponds to the increase of $d$.

Fig. 5. Dependence of the normalized frequencies on $1 / \zeta=\rho_{\mathrm{e}} / \rho_{\mathrm{L}}=\rho_{\mathrm{e}} / \rho_{\mathrm{R}}$ for $E=7-$ $3 \sqrt{5} \approx 0.292$. The left and right panels correspond to $\lambda=0$ and $\lambda=1$. The dashed and dotted curves correspond to the high-frequency and low-frequency eigenmodes. The frequencies of the fundamental mode, and the first and second overtones are shown. have to be mirror symmetric with respect to the $y$-direction, otherwise their directions are arbitrary.

Van Doorsselaere et al. (2008) pointed out that the degeneracy of kink oscillations is related to the use of the long wavelength approximation. The account of dispersion related to the finite thickness of the tubes would remove it. This statement clearly follows from the numerical results obtained by Luna et al. (2008). These authors studied kink oscillations of the two-tube system with the tubes having the same radii and the density inside the tubes being equal. They obtained that there are four different modes of kink oscillations of this system. The polarization in these modes corresponds either to $\sigma_{0}=0$, so that the tube displacements are in the $x$-direction, or to $\sigma_{0}=\pi / 2$, so that the tube displacements are in the $y$-direction. Luna et al. (2008) denoted these modes as $S_{x}$ and $A_{x}$, and as $S_{y}$ and $A_{y}$ respectively, where $S$ and $A$ stay for symmetric and antisymmetric.

In the symmetric modes the two tubes oscillate in phase, while in the antisymmetric modes they oscillate in anti-phase. The frequencies of all four modes are different, however the frequencies of $S_{x}$ and $A_{y}$ modes are smaller than the frequencies of $A_{x}$ and $S_{y}$ modes. In fact, the frequencies of $S_{x}$ and $A_{y}$ modes are close to $\omega_{-}$, and the frequencies of $S_{y}$ and $A_{x}$ modes are close to $\omega_{+}$.

As we have already mentioned Luna et al. (2008) considered the system of two identical magnetic tubes. Van Doorsselaere et al. (2008) found that the modes $S_{x}, A_{x}, S_{y}$ and $A_{y}$ exist only for a definite range of parameters corresponding to the standard systems in their terminology. There are also the so-called anomalous systems. In an anomalous system there are two $A_{x}$ modes, one with the high and one with the low frequency, and two $S_{y}$ modes, once again one with the high and one with the low frequency. There are no $A_{y}$ and $S_{x}$ modes. All systems with equal densities inside the tubes are standard systems.

In our calculations we always took the value of parameters that correspond to standard systems when the tubes are non-stratified, so that we anticipated that the system would be standard. The numerical results confirmed this conjecture. We found that $\eta_{\mathrm{R}} / \eta_{\mathrm{L}}<0$ for the smaller frequency, and $\eta_{\mathrm{R}} / \eta_{\mathrm{L}}>0$ for the larger frequency. Then it follows from Eq. (52) that, in the modes with the smaller frequency, $\boldsymbol{\xi}_{\mathrm{L}}$ and $\boldsymbol{\xi}_{\mathrm{R}}$ have the same direction when $\sigma_{0}=0$, and the opposite directions when $\sigma_{0}=\pi / 2$. This corresponds to the $S_{x}$ and $A_{y}$ modes. On the other hand, in the modes with the larger frequency, $\boldsymbol{\xi}_{\mathrm{L}}$ and $\boldsymbol{\xi}_{\mathrm{R}}$ have the opposite directions when $\sigma_{0}=0$, and the same direction when $\sigma_{0}=\pi / 2$. This corresponds to the $A_{x}$ and $S_{y}$ modes. We see that, similar to the standard system with non-stratified tubes, the system with stratified tubes have the $S_{x}$ and $A_{y}$ modes oscillating with the smaller frequency, and the $A_{x}$ and $S_{y}$ modes oscillating with the larger frequency.

\section{Implication on coronal seismology}

Verwichte et al. (2004) reported two cases of observations of the transverse coronal loop oscillations where, in addition to the fundamental harmonic, the first overtone was also observed. A very important property of these observations was that the ratio of the frequencies of the first overtone and the fundamental harmonic was less than 2. It was equal to 1.81 and 1.64 respectively (note that later Van Doorsselaere et al. (2007) used the improved technique to correct this values to 1.82 and 1.58).

Andries et al. (2005b) suggested to use these observations to estimate the scale height in the solar corona. They assumed that an oscillating loop has the half-circle shape and it is in the vertical plane. They also assumed that the atmosphere is isothermal. In that case the dependence of plasma densities inside and outside the loop are given by the expressions similar to Eq. (61). Then they calculated the ratio of frequencies of the first overtone of the loop kink oscillation to the fundamental harmonic. It turns out that this ratio is a monotonically decreasing function of $\lambda=R / H$. Hence, if we know the ratio of frequencies and 
$R$, which is the loop height at the apex point, then we can determine $H$. Using the observational data reported by Verwichte et al. (2004), Andries et al. (2005b) found $H=68 \mathrm{Mm}$ in the first event, and $H=36 \mathrm{Mm}$ in the second event.

At present there are hot debates about the internal structure of coronal loops. Two competitive models were suggested. In the first model coronal loops are considered as monolithic, while in the second as consisting of a large number of thin threads with different densities and temperatures. Andries et al. (2005b) considered loops as monolithic. Will the estimates of the coronal scale height change if the multi-thread model of coronal loops is used?

To have the first insight in this problem we can use the loop model that consists of just two threads. Let us consider the two magnetic tubes in the model studied in this paper not as separate loops, but as two threads of the same loop. We assume that the threads are in two parallel vertical planes and have the same shape, so that the equilibrium is similar to one shown in Fig. 1. We also assume that the threads oscillate in phase. Then, in accordance with the analysis in the previous section, they oscillate in the horizontal direction with the frequency $\omega_{-}$, and in the vertical direction with the frequency $\omega_{+}$.

Let us assume that the densities inside the threads are proportional to the density in the external plasma, so that $\zeta_{\mathrm{L}}$ and $\zeta_{\mathrm{R}}$ are constant. Then, introducing new variables,

$\eta_{+}=2 E\left(\zeta_{\mathrm{R}}-1\right) \eta_{\mathrm{R}}+\left(Q-\zeta_{\mathrm{L}}+\zeta_{\mathrm{R}}\right) \eta_{\mathrm{L}}$

$\eta_{-}=\left(Q-\zeta_{\mathrm{L}}+\zeta_{\mathrm{R}}\right) \eta_{\mathrm{R}}-2 E\left(\zeta_{\mathrm{L}}-1\right) \eta_{\mathrm{L}}$

we reduce the system of Eqs. (59) and (60) to

$4 V_{\mathrm{Ae}}^{2} \frac{\mathrm{d}^{2} \eta_{ \pm}}{\mathrm{d} z^{2}}+\omega^{2}\left(\zeta_{\mathrm{L}}+\zeta_{\mathrm{R}}+2 \mp Q\right) \eta_{ \pm}=0$.

We see that now we have two separate equations, one for $\eta_{+}$, and one for $\eta_{-}$. Using Eq. (52) it is not difficult to show that, in the case when the threads oscillate in phase, $\eta_{\text {- corresponds }}$ to the horizontal, and $\eta_{+}$to the vertical oscillations. Using the dimensionless quantities

$\bar{z}=\frac{z}{L}, \quad \Omega_{ \pm}^{2}=\frac{\omega^{2} L^{2}}{4 \bar{V}_{\mathrm{Ae}}^{2}}\left(\zeta_{\mathrm{L}}+\zeta_{\mathrm{R}}+2 \mp Q\right)$,

we reduce Eq. (64) to

$\frac{\mathrm{d}^{2} \eta_{ \pm}}{\mathrm{d} \bar{z}^{2}}+q(\bar{z}) \Omega_{ \pm}^{2} \eta_{ \pm}=0$

where $q(\bar{z})=\rho_{\mathrm{e}} / \bar{\rho}_{\mathrm{e}}, \bar{\rho}_{\mathrm{e}}=\rho_{\mathrm{e}}(0)$. This equation together with the line-tying condition $\eta_{ \pm}( \pm 1 / 2)=0$ constitutes the eigenvalue problem that determines the eigenfrequencies of oscillations. We see that, in dimensionless variables, the eigenvalue problems for the high-frequency and low-frequency oscillations are the same. Moreover, Dymova \& Ruderman (2005) showed that the kink oscillations of a thin straight magnetic tube in a cold plasma with the density varying along the tube are governed by the equation

$\frac{\mathrm{d}^{2} \eta}{\mathrm{d} z^{2}}+\frac{\omega^{2}}{C_{k}^{2}} \eta=0, \quad C_{k}^{2}=\frac{2 B^{2}}{\mu_{0}\left(\rho_{\mathrm{i}}+\rho_{\mathrm{e}}\right)}$,

where $\rho_{\mathrm{i}}(z)$ and $\rho_{\mathrm{e}}(z)$ are the densities inside and outside the tube. If $\rho_{\mathrm{i}} / \rho_{\mathrm{e}}=\zeta=$ const, then, in the dimensionless variables

$\bar{z}=\frac{z}{L}, \quad \Omega^{2}=\frac{\omega^{2} L^{2}(\zeta+1)}{2 \bar{V}_{\mathrm{Ae}}^{2}}$,
Eq. (67) is transformed to

$\frac{\mathrm{d}^{2} \eta}{\mathrm{d} \bar{z}^{2}}+q(\bar{z}) \Omega^{2} \eta=0$

We see that Eq. (69) coincides with Eq. (66). Let $\Omega_{\mathrm{f}}$ be the fundamental frequency and $\Omega_{\mathrm{on}}, n=1,2, \ldots$ are the frequencies of overtones of the eigenvalue problem that consists of Eq. (66) (or, which is the same, Eq. (69)) and the boundary conditions $\eta( \pm 1 / 2)=0$. Then the squares of the dimensional frequencies of the high-frequency and low-frequency kink oscillations of the two thread system, and the monolithic loop, are given by

$\omega_{\mathrm{f}+}^{2}=\frac{4 \Omega_{\mathrm{f}}^{2} \bar{V}_{\mathrm{Ae}}^{2} L^{-2}}{\zeta_{\mathrm{L}}+\zeta_{\mathrm{R}}+2-Q}, \quad \omega_{\mathrm{on}+}^{2}=\frac{2 \Omega_{\mathrm{on}}^{2} \bar{V}_{\mathrm{Ae}}^{2} L^{-2}}{\zeta_{\mathrm{L}}+\zeta_{\mathrm{R}}+2-Q}$,

$\omega_{\mathrm{f}-}^{2}=\frac{2 \Omega_{\mathrm{f}}^{2} \bar{V}_{\mathrm{Ae}}^{2} L^{-2}}{\zeta_{\mathrm{L}}+\zeta_{\mathrm{R}}+2+Q}, \quad \omega_{\mathrm{on}-}^{2}=\frac{2 \Omega_{\mathrm{on}}^{2} \bar{V}_{\mathrm{Ae}}^{2} L^{-2}}{\zeta_{\mathrm{L}}+\zeta_{\mathrm{R}}+2+Q}$,

$\omega_{\mathrm{f}}^{2}=\frac{2 \Omega_{\mathrm{f}}^{2} \bar{V}_{\mathrm{Ae}}^{2} L^{-2}}{\zeta+1}, \quad \omega_{\mathrm{on}}^{2}=\frac{2 \Omega_{\mathrm{on}}^{2} \bar{V}_{\mathrm{Ae}}^{2} L^{-2}}{\zeta+1}$,

where the indices "f" and "o" indicate the fundamental mode and overtones, and $n=1,2, \ldots$ is the ordinal number of an overtone. We see that the frequencies of the horizontal and vertical oscillations of the two thread system, and the frequencies of kink oscillations of a monolithic loop are different. This difference is caused by the fact that the non-stationary flow of the external plasma around an oscillating monolithic loop is different from that around an oscillating two thread system. However, the ratios of frequencies of overtones to the fundamental frequency are the same. This means that the estimate of the scale height obtained by Andries et al. (2005b) would not change if we assume that the observed kink oscillations were the oscillations of the loop consisting of two threads.

Andries et al. (2005b) took $\zeta=10$ in their numerical investigation. It follows from our analysis that the ratios of the frequencies of the overtones and the fundamental mode is independent of $\zeta$. The only condition that has to be satisfied is that $\zeta=$ const.

\section{Summary and conclusions}

In this paper we studied the kink oscillations of two parallel coronal loops with the density varying along the loop. We modelled this two-loop system by the system of two parallel magnetic tubes. Using bicylindrical coordinates we derived the system of equations governing the oscillations of the two-tube system. We then have used this system to study the eigenmodes of kink oscillations. First we re-derived the expressions for the frequencies of kink oscillations of two homogeneous parallel tubes previously derived by Van Doorsselaere et al. (2008). Then we considered the effect of density variation along the tubes. We assumed that the loops are in two parallel vertical planes and have the shape of half-circles with the same radius $R$. We also assumed that the atmosphere is isothermal and the plasma temperature is the same in the loops and in the surrounding plasma. This implies that the ratios of plasma densities inside the loops to the density of the external plasma are constant. The eigenvalue problem describing the eigenfrequencies of the loop oscillations was solved numerically.

We investigated the implication of our results on coronal seismology. When doing so we concentrated on the problem of determination of the atmospheric scale hight using the ratio of 
frequencies of the fundamental harmonic and first overtone of the kink oscillations. We compared the results obtained on the basis of two models: one where a coronal loop is assumed to be monolithic, and the other where a coronal loop consists of two threads. We assumed that the ratios of plasma densities inside the loops and in the external plasma are constant. Then we showed that, under this assumption, the two models give exactly the same value of the atmospheric scale height. Moreover, we showed that this value is independent of the density ratios.

Acknowledgements. M.S.R. and Y.T. acknowledge the support by an STFC grant. D.R. acknowledges the support by an STFC postgraduate fellowship.

\section{References}

Andries, J., Goossens, M., Hollweg, J. V., Arregui, I., \& Van Doorsselaere, T. 2005a, A\&A, 430, 1109

Andries, J., Arregui, I., \& Goossens, M. 2005b, ApJ, 624, L57

Andries, J., van Doorsselaere, T., Roberts, B., et al. 2009, Space. Sci. Rev., 149, 3
Aschwanden, M. J., Fletcher, L., Schrijver, C. J., \& Alexander, D. 1999, ApJ, 520,880

Diaz, A. J., Oliver, R., \& Ballester, J. L. 2006, ApJ, 645, 766

Dymova, M. V., \& Ruderman, M. S. 2005, Solar Phys., 229, 79

Dymova, M. V., \& Ruderman, M. S. 2006a, A\&A, 457, 1059

Dymova, M. V., \& Ruderman, M. S. 2006b, A\&A, 459, 241

Edwin, P. M., \& Roberts, B. 1983, Solar Phys., 88, 179

Korn, G., \& Korn, T. 1961, Mathematical handbook for scientists and engineers (New York: McGraw-Hill)

Luna, M., Terradas, J., Oliver, R., \& Ballester, J. L. 2008, ApJ, 676, 717

Luna, M., Terradas, J., Oliver, R., \& Ballester, J. L. 2009, ApJ, 692, 1582

McEwan, M. P., Diaz, A. J., \& Roberts, B. 2008, A\&A, 481, 819

Morton, R. J, \& Erdelyi, R. 2009, A\&A, 502, 315

Nakariakov, V. M., Ofman, L., DeLuca, E. E., Roberts, B., \& Davila, J. M. 1999, Science, 285, 862

Ofman, L. 2005, Adv. Space Res., 36, 1572

Ofman, L. 2009, ApJ, 694, 502

Ruderman, M. S., \& Erdélyi, R. 2009, Space. Sci. Rev., 149, 199

Van Doorsselaere, T., Nakariakov, V. M., \& Verwichte, E. 2007, A\&A, 473, 959

Van Doorsselaere, T., Ruderman, M. S., \& Robertson, D. 2008, A\&A, 485, 849

Verwichte, E., Nakariakov, V. M., Ofman, L., \& Deluca, E. E. 2004, Solar Phys., 223, 77 